\title{
Improving the Efficiency of a Coagulation-Flocculation Wastewater Treatment of the Semiconductor Industry through Zeta Potential Measurements
}

\author{
Eduardo Alberto López-Maldonado, ${ }^{1}$ Mercedes Teresita Oropeza-Guzmán, ${ }^{1,2}$ \\ and Adrián Ochoa-Terán ${ }^{2}$ \\ ${ }^{1}$ Centro de Investigación y Desarrollo Tecnológico en Electroquímica (CIDETEQ), Carretera Libre Tijuana-Tecate km. 26.5, \\ Parque Industrial El Florido, 22500 Tijuana, BC, Mexico \\ ${ }^{2}$ Centro de Graduados e Investigación en Química del Instituto Tecnológico de Tijuana, Boulevard Alberto Limón Padilla s/n, \\ Mesa de Otay, 22500 Tijuana, BC, Mexico
}

Correspondence should be addressed to Eduardo Alberto López-Maldonado; eduardo_a_lm@hotmail.com

Received 6 November 2013; Revised 11 June 2014; Accepted 12 June 2014; Published 10 July 2014

Academic Editor: Saima Q. Memon

Copyright (C) 2014 Eduardo Alberto López-Maldonado et al. This is an open access article distributed under the Creative Commons Attribution License, which permits unrestricted use, distribution, and reproduction in any medium, provided the original work is properly cited.

\begin{abstract}
Efficiency of coagulation-flocculation process used for semiconductor wastewater treatment was improved by selecting suitable conditions $(\mathrm{pH}$, polyelectrolyte type, and concentration) through zeta potential measurements. Under this scenario the zeta potential, $\zeta$, is the right parameter that allows studying and predicting the interactions at the molecular level between the contaminants in the wastewater and polyelectrolytes used for coagulation-flocculation. Additionally, this parameter is a key factor for assessing the efficiency of coagulation-flocculation processes based on the optimum dosages and windows for polyelectrolytes coagulation-flocculation effectiveness. In this paper, strategic $\mathrm{pH}$ variations allowed the prediction of the dosage of polyelectrolyte on wastewater from real electroplating baths, including the isoelectric point (IEP) of the dispersions of water and commercial polyelectrolytes used in typical semiconductor industries. The results showed that there is a difference between polyelectrolyte demand required for the removal of suspended solids, turbidity, and organic matter from wastewater $(23.4 \mathrm{mg} / \mathrm{L} \mathrm{and} 67 \mathrm{mg} / \mathrm{L}, \mathrm{resp}$.). It was also concluded that the dose of polyelectrolytes and coagulation-flocculation window to achieve compliance with national and international regulations as EPA in USA and SEMARNAT in Mexico is influenced by the physicochemical characteristics of the dispersions and treatment conditions ( $\mathrm{pH}$ and polyelectrolyte dosing strategy).
\end{abstract}

\section{Introduction}

In the industrial wastewater treatment of semiconductors, the most appreciated characteristic of polyelectrolytes (PE) is their solid-liquid separation efficiency, with extensive application in purification of drinking water, industrial raw and process water, municipal sewage treatment, mineral processing and metallurgy, oil drilling and recovery, paper and board production, and so forth [1-9]. Polyelectrolytes can be used alone or in association with other flocculant aids, such as inorganics salts, surfactants, or even as a second polymer. Compared with inorganic coagulants, there are some advantages by using organic polyelectrolytes $[1,6,7,10$, 11]: lower dose requirements, a smaller volume of sludge, a smaller increase in the ionic load of the treated water, and cost savings up to $25-30 \%$.

It is well known that the efficiency of a certain polyelectrolytes in flocculation processes is evaluated as a function of four main parameters: the optimum flocculant concentration, which should be as low as possible; isoelectric point (IEP) that determines the effective $\mathrm{pH}$ range; and the flocculation window, which must be as large as possible [12]. To improve the solid/liquid separation process, polymeric flocculants, mainly polycations, have been used $[1,6-9]$. The main 
disadvantage of flocculation with polymers is the very small flocculation window, risking particles resuspension with few dosage increases.

A number of studies have tried to solve this problem by combining two or more oppositely charged polyelectrolytes that can be added one after another [13-16] or as nonstoichiometric polyelectrolyte complexes (NIPECs) [1724]. In the first case, a combination of a low molecular cationic weight and a high-molecular weight anionic polymer produce synergism during flocculation. This system is known as "dual flocculation process" [25]. For fine and ultrafine solid suspensions, use of double flocculant systems seems to offer a promising route for enhanced solid-liquid separation [25]. The dosing sequence, polymer size, and charge density all affect flocculation significantly. Enhanced flocs form through a combination of oppositely charged polyelectrolyte under suitable condition $[13,14,22,26,27]$.

The investigations on NIPECs flocculants, as colloidal dispersions bearing positive or negative charges in excess, which started with the preliminary studies of Kashiki and Suzuki $[17,18]$ and were developed in the last years [1924], have been concentrated on the use of NIPECs with molar ratio between charges ranged from 0.4 to 0.8 . The main advantage in flocculation induced by NIPECs is the lower dependence on the concentration of the flocculants, showing a substantially wider optimum concentration range. Nevertheless, the optimum concentration required for flocculation with NIPECs was found to be higher than the optimum concentration for flocculation with polycations. Dual flocculation (sequential addition of two chemicals) presents some advantages compared with single polymer flocculant, as higher overall level of aggregation, less sensitivity to variations of polyelectrolyte concentration, good sludge dewatering, superior retention, and shear-resistant flocs, and so forth. All these issues improve control and optimization maneuvers of the flocculation process.

As mentioned above, in the semiconductor assembly industry the wastewater treatment system consists of a coagulation-flocculation process in which anionic and cationic polyelectrolytes are used to remove suspended solids, organic matter, and cation content so that the effluent meets the maximum permissible limits $\mathrm{Pb} 1 \mathrm{ppm}$, Ni $3.0 \mathrm{ppm}, \mathrm{Cu}$ $1.2 \mathrm{ppm}$, biochemical oxygen demand $\left(\mathrm{BOD}_{5}\right) 60 \mathrm{mg} \mathrm{O} / \mathrm{L}$, chemical oxygen demand (COD) $150 \mathrm{mg} \mathrm{O}_{2} / \mathrm{L}$, and total nitrogen (TN) $25 \mathrm{mg} / \mathrm{L}$, prior to being discharged into the municipal sewer system and/or turned to be reused as service water in cooling towers, heat exchangers, and production.

One of the key stages in the production line of semiconductors is electroplating (EP). Its wastewater contains high concentration of suspended solids, organic compounds, and dissolved cation. Under these conditions, polyelectrolyte dosing should guarantee the entire maximum permissible concentration. Commonly operators in the wastewater treatment plant are used to modifying any independent variable as $\mathrm{pH}$ and $\mathrm{PE}$ dose to achieved the maximum allowable limit in total suspended solids (TSS) and cation content; however, the treated water is affected by another important parameter as $\mathrm{COD}$, total organic carbon (TOC), and $\mathrm{BOD}_{5}$.
In this work the authors decided to use real wastewater coming from the EP process, considering this water as the greater challenges for solid-liquid separation in the semiconductor industry. First of all, traditional coagulationflocculation windows construction was followed with zeta potential measurements as well as turbidity, TSS, COD, and TOC to demonstrate that solid-liquid separation can be predicted by zeta potential measurements and could help to improve the coagulation-flocculation process efficiency, knowing the optimal polyelectrolyte dosage and opening a potential way for polyelectrolytes design ensuring low environmental impact of polyelectrolyte overdose.

\section{Experimental}

The experimental work was made in three steps. The first one was the characterization of electroplating raw wastewater under the Mexican environmental regulations NOM-002SEMARNAT-1997 (Zn 1.2 ppm, Ni 3.0 ppm, Cu 1.2 ppm, Pb $1.0 \mathrm{ppm}$, TN $25 \mathrm{mg} \mathrm{N} / \mathrm{L}$, TSS $60 \mathrm{ppm}$, COD $150 \mathrm{mg} \mathrm{O}$ /L, and $\mathrm{BOD}_{5} 60 \mathrm{mg} \mathrm{O} / \mathrm{L}$ ), the second was to construct the $\mathrm{pH}-\zeta$ diagrams allowed to establish different polyelectrolyte dosing strategies, and the third constructs coagulation-flocculation windows.

The experimental strategy followed to study the conditions of coagulation-flocculation process was to examine the profiles of $\mathrm{pH}$ and $\zeta$ of commercial polyelectrolytes used in assembly semiconductor wastewater treatment plant (WWTPs). Once the isoelectric point of the coagulant and flocculant was determined, as well as the sampled wastewater, polyelectrolytes dosing was studied at different $\mathrm{pH}$ values. In parallel the effect of using an interpolyelectrolyte complex flocculation in the window was tested. Finally, the effect of coagulant dose in the flocculation window by the dual flocculation process was performed. In Figure 1 the experimental methodology to evaluate the physicochemical performance of $\mathrm{PE}$ in coagulation-flocculation windows is resumed.

2.1. Materials. Polydadmac (OPTIFLOC C-1008) and flocculant (Trident 27,506) are commercial polyelectrolytes used in a semiconductor industry.

All reagents used in testing water quality $(\mathrm{COD}, \mathrm{TN}$, TOC, and $\mathrm{BOD}_{5}$ ) were obtained from Hach as follows:

Digesdahl Hach (microKendahl digestion apparatus), Hach Digital Reactor DRB200 (Digestor),

Denver Instrument pH UP-5 (Potentiometer),

DR/890 Hach (Colorimeter),

HQ40d Multi Hach (measuring equipment for dissolved oxygen and conductivity),

Dissolved Oxygen Meter (Luminescent Dissolved Oxygen) LD0101-01 Probe,

Conductivity Meter CDC401-01 Probe,

Atomic Absorption Spectrophotometer GBC 932 Plus,

Zetasizer Nano-ZS, model ZEN3500. 


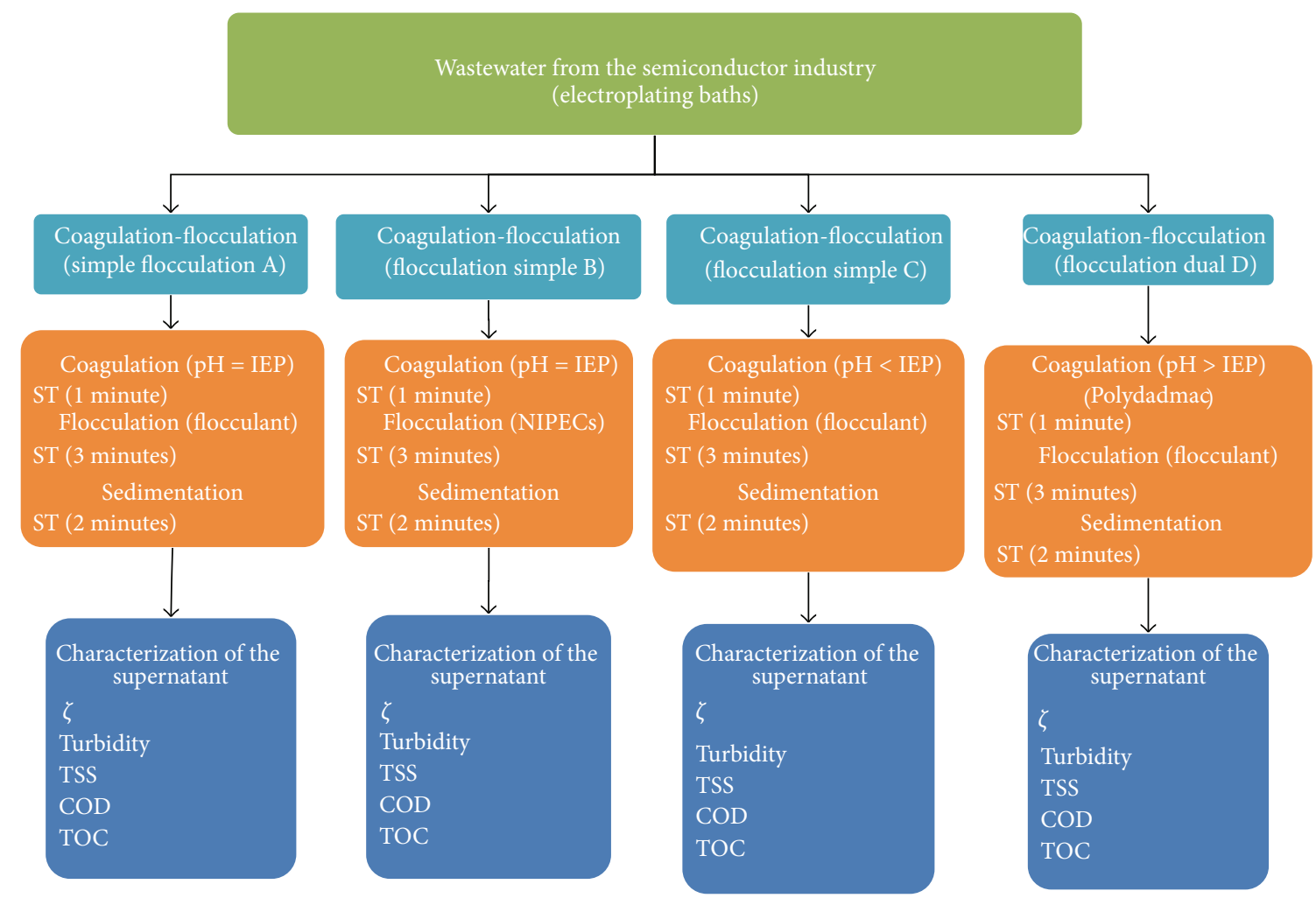

FIGURE 1: Experimental conditions for the physicochemical evaluation of polyelectrolytes used in coagulation-flocculation processes for electroplating wastewater in semiconductor industry.

\subsection{Methods}

2.2.1. Residual Water Sampling in the Assembling Semiconductor Industry. The wastewater sampling protocol in the assembling semiconductor industry was followed as recommended by sampling Mexican standards (NMX-AA-003-1980).

2.2.2. Preparation of the Dispersion of Wastewater. The wastewater dispersions were prepared diluting $5 \mathrm{~mL}$ or raw wastewater in deionized water using a volumetric flask of $50 \mathrm{~mL}$. Since the electroplating process is in continuous operation and following a timetable protocol, the sampled water content is considered as reproducible.

2.2.3. Preparation of NIPECs. Anionic NIPECs synthetic solutions were prepared in relationships between 0.1 to $2 \mathrm{mg}$ of cationic PE/mg anionic PE. Each solution was stirred before $\zeta$ measurement.

2.2.4. Colloid Titration. Polydadmac $10 \mathrm{ppm}$ was prepared taking a certain amount of a solution of $1.28 \mathrm{mM}$ of polydadmac and diluting in $10 \mathrm{~mL}$ with distilled water in a volumetric flask. The prepared solution was poured into a $20 \mathrm{~mL}$ vial and measured to be entitled initial zeta potential. Then a certain amount of titrant solution $1.24 \mathrm{mM}$ poly (vinyl sulfate) potassium salt (PVSK) was added. Each solution was stirred before measurement. Various additions of titrant were made to reach the isoelectric point (turbidity appears in solution).
2.2.5. Micro-Jar Tests. A sample of $5 \mathrm{~mL}$ of wastewater electroplating process was diluted with $50 \mathrm{~mL}$ of deionized water into a volumetric flask. $\mathrm{pH}$ was adjusted (5, 7, and 9). A second solution of wastewater with adjusted $\mathrm{pH}$ was prepared using a dilution factor of 0.5 . The polyelectrolyte dosage tests (micro-Jar tests) were performed in $20 \mathrm{~mL}$ vials. Progressive additions of flocculant solution $0.1093 \mathrm{~g} / \mathrm{L}$ were done and after each one, the vials were shaken for $2 \mathrm{~min}$ and allowed to settle for 2 more minutes. Finally, the supernatant was suctioned to determine turbidity, $\zeta$, TSS, TOC, and COD.

2.2.6. Analytical Techniques. Immediately, the samples were tested for $\mathrm{BOD}_{5}, \mathrm{COD}$, and TOC, five composite samples were analyzed following the procedures and test methods established by Mexican standards (NMX-AA-028-SCFI-2001, NMX-AA-030-SCFI-2001).

\section{Results and Discussions}

3.1. Characterization of Wastewater from Electroplating Process in a Semiconductor Industry. The analysis of wastewater from different process units, in the assembling semiconductor industry, showed that electroplating section is the more challenging one. However, a detailed chemical composition is uncertain due to the large amount of chemical mixtures employed in the electroplating baths [28]. As shown in Table 1 electroplating wastewater has a high COD, turbidity, and total dissolved solids as well as $\mathrm{Pb}$ and $\mathrm{Sn}$ concentration. 
TABLE 1: Characterization of electroplating wastewater.

\begin{tabular}{lc}
\hline Parameter & ppm \\
\hline $\mathrm{Sn}$ & 4854 \\
$\mathrm{~Pb}$ & 1044 \\
$\mathrm{Fe}$ & 683 \\
$\mathrm{TSS}$ & 4510 \\
Turbidity (FAU) & 2990 \\
$\mathrm{EC}(\mathrm{mS} / \mathrm{cm})$ & 74 \\
$\zeta(\mathrm{mV})$ & 45 \\
$\mathrm{Size}(\mathrm{nm})$ & 346 \\
$\mathrm{Color}$ & $\mathrm{Milky}$ \\
$\mathrm{pH}$ & 0.8 \\
$\mathrm{COD}\left(\mathrm{mg} \mathrm{O}_{2} / \mathrm{L}\right)$ & 1432 \\
$\mathrm{TOC}\left(\mathrm{mg} \mathrm{C}_{\mathrm{L}}\right)$ & 125 \\
$\mathrm{BOD}_{5}\left(\mathrm{mg} \mathrm{O}_{2} / \mathrm{L}\right)$ & 30 \\
$\mathrm{TN}\left(\mathrm{mg} \mathrm{N}^{\mathrm{L}}\right)$ & 50.6 \\
Biodegradability $\left(\mathrm{BOD}_{5} / \mathrm{COD}\right)$ & 0.04 \\
\hline
\end{tabular}

Due to the fact that coagulation-flocculation processes are used in the installed WWTPs, the key parameters to follow-up are turbidity, TSS, and zeta potential $(\zeta)$. All of them are directly related to the stability of the suspended solids. For raw wastewater $\zeta=45 \mathrm{mV}(\mathrm{pH}=0.8)$, indicating the presence of positively charged particles suspended in water and probably related to metallic cations adsorbed on particles surface. For particles of $346 \mathrm{~nm}$, longer sedimentation times are expected. Physical properties as turbidity and TSS also indicate that raw wastewater is a stable dispersion. Considering water properties, the solids separation using coagulation-flocculation processes seems to require the addition of negative polyelectrolytes. However, the specific dose is unknown as is the real effect of negative polyelectrolyte addition on the solid separation. In this paper, traditional coagulation-flocculation process is analyzed to determine the optimal polyelectrolyte dose as well as the more suitable $\mathrm{pH}$ condition to improve the efficiency and to diminish the environmental impact caused by polyelectrolyte overdose.

3.2. Determination of the Isoelectric Point for the Electroplating Wastewater. In the first stage (coagulation) suspended solids were destabilized by changing the water $\mathrm{pH}$ until isoelectric point is approached. A simple $\mathrm{pH}$ variation could be enough to stabilize or destabilize dispersions. Moreover, the performance of polymeric $\mathrm{PE}$ is influenced by the wastewater $\mathrm{pH}$. Thus, the $\mathrm{pH}$ value may control both polyelectrolyte charge density and suspended particles surface charge. The isoelectric point of the dispersion generated in the electroplating process will be detected in a plot of $\zeta$ versus $\mathrm{pH}$.

Using the colloidal titration method (with $\zeta$ as detection point) the charge density (CD) of the anionic and cationic PE (flocculant and coagulant) was determined: polydadmac $22 \mathrm{meq} / \mathrm{g}$ and flocculant $5 \mathrm{meq} / \mathrm{g}$. These values confirm why polydadmac is usually a coagulant due to its high charge density, while the flocculant does not require high $\mathrm{CD}$ to accomplish the solid agglomeration. In Figure 2, the plot $\zeta$

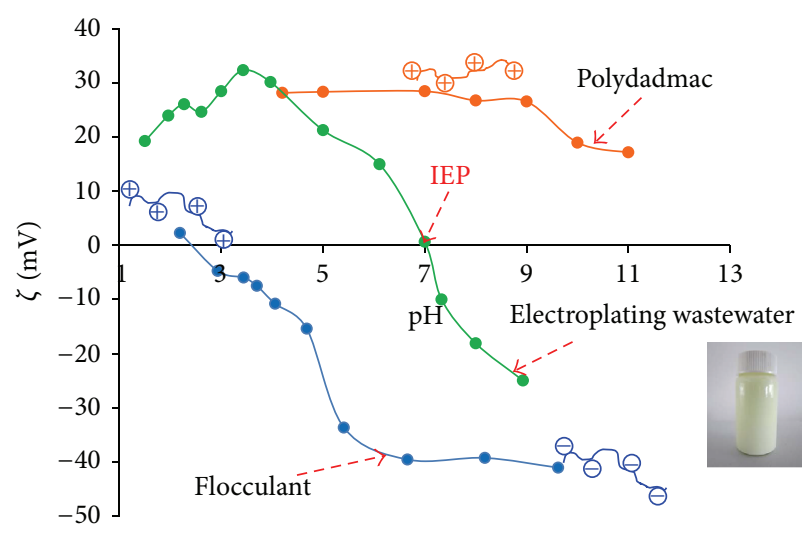

FIGURE 2: Electrokinetic properties of the dispersion of wastewater from electroplating, $20.8 \mathrm{ppm}$ polydadmac, and $21.9 \mathrm{ppm}$ flocculant.

versus $\mathrm{pH}$ shows the $\mathrm{CD}$ variation for suspended particles in wastewater, as well as the proper $\mathrm{pH}$ to achieve the isoelectric point. In the same plot, it can be observed that polydadmac does not reach an isoelectric point and flocculant has one at very low $\mathrm{pH}$. This result implies that at $\mathrm{pH}>5$, flocculant is expected to be very efficient, while polydadmac is effective at $\mathrm{pH}<10$ (diminish of its $\mathrm{CD}$ is certainly due to the ammonium hydrolysis).

To corroborate the eye observable phenomena (TSS) with the interfacial phenomena (zeta potential) and nanoscale particle diameters, different combinations were studied and are presented below. Further studies were conducted to analyze the influence of $\mathrm{PE}$ dosage with organic matter content (COD and TOC).

The coagulation-flocculation experiments are generally divided into two parts: single flocculation (optimum condition for operation using only one PE) and dual PE flocculation.

3.3. Single Flocculation (A). $\mathrm{pH}$ adjustment was used to reach the isoelectric point ( $\mathrm{pH} 7$ ); however, the settling kinetics was too slow and the flocculant addition was needed. Even if turbidity and TSS are the most common physical properties that guide the water clarification, they are not recommended for controlling dosage or investigate the source of an operation problem. To show this fact, in Figure 3 turbidity (FAU), TSS, $\zeta$, electric conductivity, TOC, and COD were plotted as a function of flocculant dosage in single flocculation of the neutralized wastewater. In these plots three main regions are identified: low dose, where at the flocculant concentration in the residual water does not allow the wastewater clarification; the optimal dose region, which corresponds to the optimum flocculant concentration for the total removal of TSS and turbidity; and the overdose region, indicating flocculant concentrations that cause stabilization of the dispersed particles and have an adverse effect on the quality of treated water. The magnitude Figure 6 of TSS and turbidity is the same (thousands) indicating that the generation of turbidity and TSS is due to the same cause. 


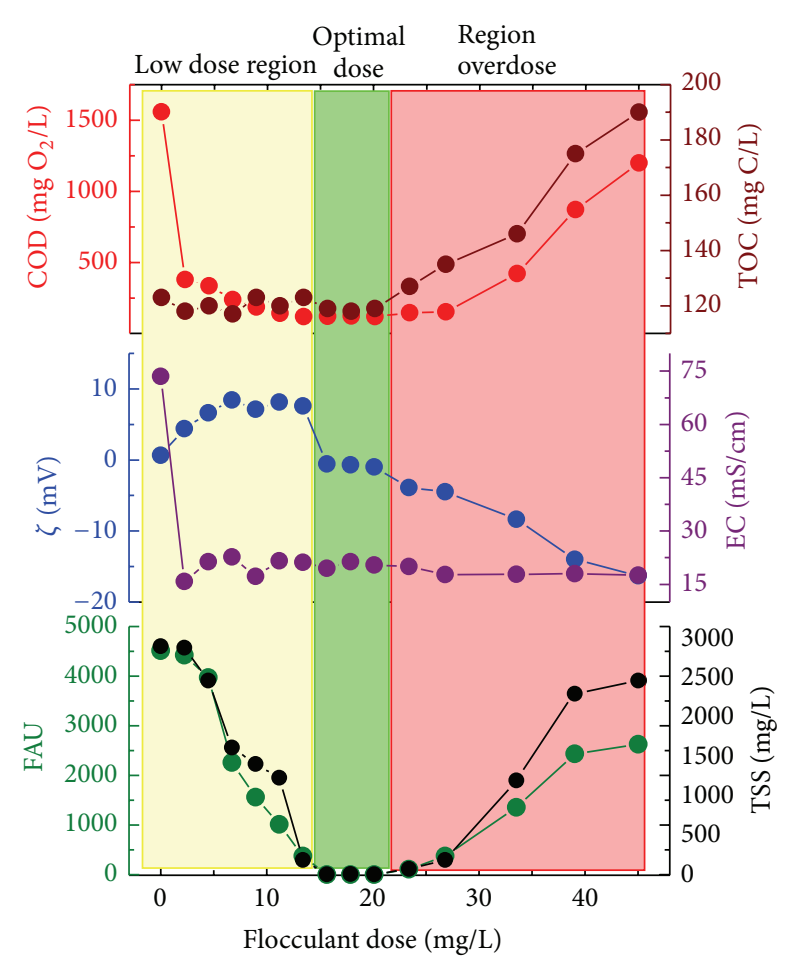

FIgUre 3: Turbidity, TSS, $\zeta$, EC, COD, and TOC of the supernatant in the single flocculation at $\mathrm{pH} 7$ with flocculant.

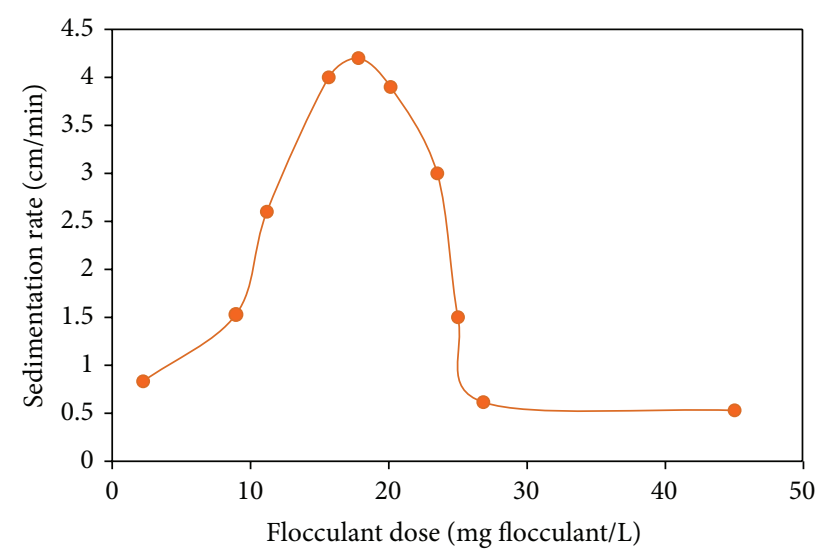

FIGURE 4: Sedimentation rate of suspended solids.

Figure 3 indicates that at low dosages $(2$ and $13 \mathrm{mg} / \mathrm{L}$ ) of flocculant, the $\zeta$ potential is approximately $8 \mathrm{mV}$ and the turbidity is high (2587 FAU). At the point where the $\zeta$ potential value becomes $-0.7 \mathrm{mV}$ ( $15.6 \mathrm{ppm}$ flocculant), the turbidity is at an absolute minimum. After this point in nearly obtaining a zero charge and turbidity, further addition of flocculating agent now reverses and increases the charge of the contaminating material $(\zeta=-16.3 \mathrm{mV}$ and turbidity $=2630 \mathrm{FAU})$ and therefore restabilizes these particles in the water. In this region of overdose, the polyelectrolyte in excess causes the adsorption of polyelectrolyte chains onto the stable particles in suspension, having an adverse effect on water quality. This is observed by increasing the

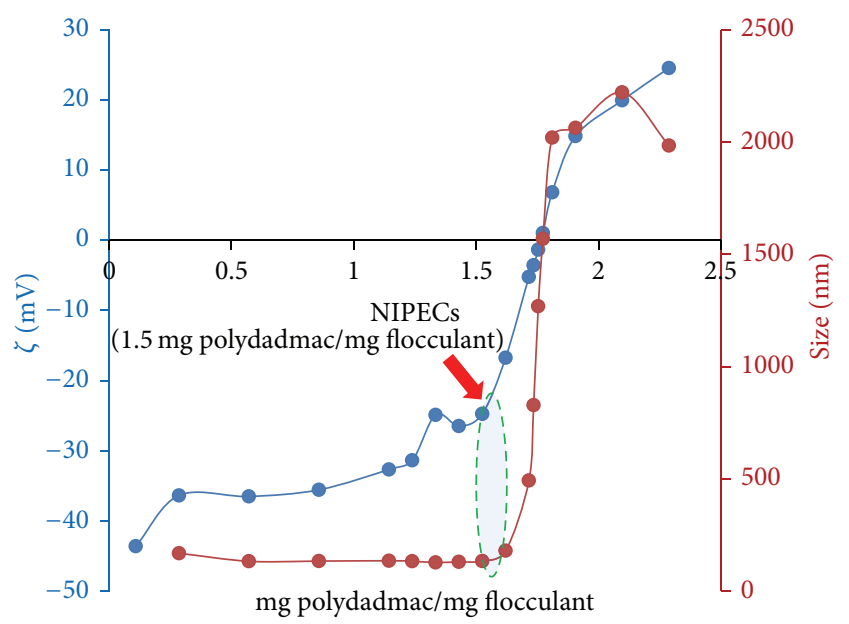

Figure 5: NIPECs of polydadmac/flocculant.
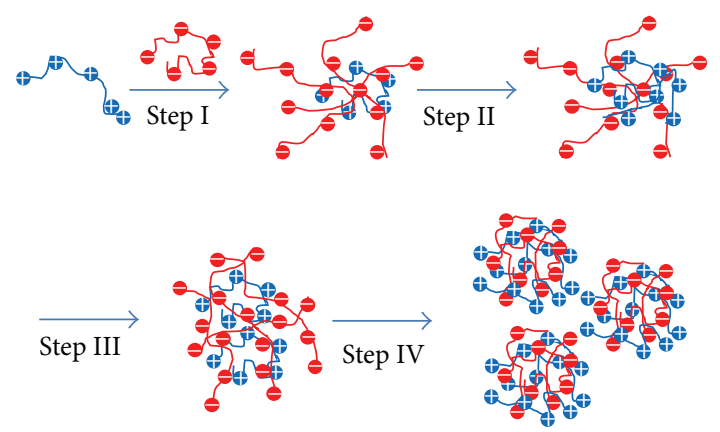

Figure 6: Proposed mechanism for the formation of NIPECs.

turbidity and suspended solids as zeta potential become more negative. The shift of the value of $\zeta$ is due to the negative charge of the flocculant. Electric conductivity of water remains virtually constant after the first additions of flocculant. For the range between 2 and $13 \mathrm{mg} / \mathrm{L}$, the supernatant has small but positive zeta potential $(8 \mathrm{mV})$. Figure 3 also shows the variation in the TOC and COD content in the supernatant. It is noted that since the first dose of flocculant (2 ppm) COD at 1500 decreases to a value of 380 ppm, subsequently, a gradual COD decrease occurs with increasing flocculant dose until the optimal dose of $15.6 \mathrm{mg} / \mathrm{L}$.

It is also noted that the TOC is almost constant in the interval of the flocculation window. At the point of optimum flocculant dose $(15.6 \mathrm{mg} / \mathrm{L})$ the supernatant has a COD equal to $122 \mathrm{mg} \mathrm{O}_{2} / \mathrm{L}$ and TOC equal to $119 \mathrm{mg} \mathrm{C} / \mathrm{L}$, which indicates that the treatment applied do not remove residual organic matter. Figure 4 shows the rate of sedimentation of suspended solids in each dose of flocculant. The relationship between flocculant dose and sedimentation speed presents a maximum. In the region of overdose, the sedimentation rate decreases by restabilization of the suspended solids. 
3.4. NIPECs Simple Flocculation (B). Various materials have been developed in recent years for coagulation and flocculation purposes, as inorganic-based coagulants, organicbased flocculants, and hybrid materials [29]. The continuous increase of market needs for more efficient and effective materials in wastewater treatment has induced the development of hybrid materials for coagulation-flocculation of wastewater. Hybrid materials thus have emerged as new materials that pose tremendous potential in treating wastewater due to their better performance compared to that of conventional inorganic-based coagulants and its lower cost than that of organic-based flocculants [30].

Due to the synergetic effect of hybrid components in one material, hybrid materials hence pose a superior performance than that of individual component [31-33]. Compared with individual coagulant/flocculant, hybrid materials, which have combined functional components into one prescription, would be a convenient alternative material for the operation of wastewater treatment facilities since the whole wastewater treatment can be conducted with the addition of one chemical and in one tank instead of two unit operations in the conventional coagulation-flocculation system. Reduction of operation time as a result of the application of these hybrid materials in a single operation is favorable to the industries that are discharging large volumes of wastewater [34].

Within the hybrid materials currently used to improve coagulation-flocculation processes are the nonstoichiometric polyelectrolyte complexes (NIPECs).

Figure 5 shows the variation of $\zeta$ and size of the colloidal particles formed according to the relationship $\mathrm{mg}$ polydadmac/mg flocculant. It can be seen that the zeta potential becomes more positive as the amount of cationic polyelectrolyte increases, while the size remains constant as far as $\mathrm{mg}$ polydadmac/mg flocculant varies from 0.9 to 1.5 . At ratios greater than 1.6, the colloidal particle size increases suddenly. The colloidal particles obtained in a ratio of 1.7 are neutral and have a size of 1.5 microns, and at this point the isoelectric point is reached. Interpolyelectrolyte complexes formed in ratios greater than 1.8 have a size of $2 \mathrm{~mm}$ and an excess of positive charge is reflected by the zeta potential value of $15 \mathrm{mV}$. During all these experiments, the dispersions characteristics remained constant indicating that changes of $\zeta$ and particle size variations were due to the NIPECs concentration and not for agglomeration kinetics.

\subsubsection{Proposed Mechanism for the Formation of NIPECs.} Taking into account the DLS and $\zeta$ potential results, a mechanism in four steps has been assumed and presented in Figure 6.

In the first step, the added polydadmac interacts with flocculant chains leading to the primary aggregates, which, taking into account the differences in the flexibility of the complementary polyions and the mismatch of charges, may contain more flocculant chains connected by fewer polydadmac chains; such aggregates would have a high density of free negative charges compensated with small counterions, not by polydadmac charges.

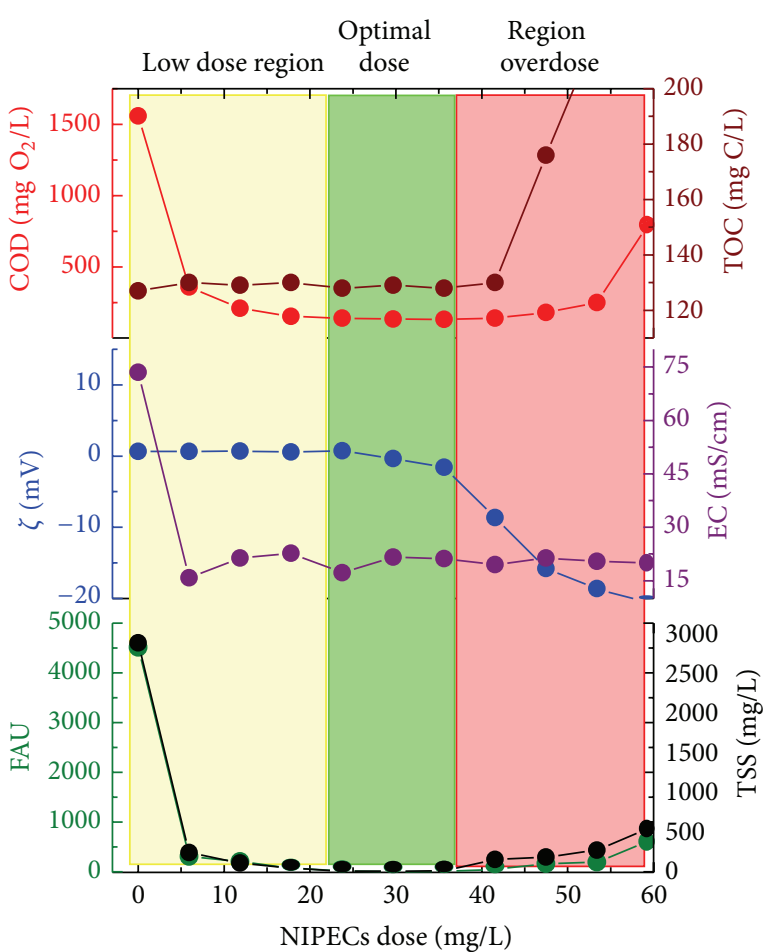

FIgUre 7: Turbidity, TSS, $\zeta$, EC, COD, and TOC of the supernatant in the single flocculation at $\mathrm{pH} 7$ with NIPECs.

The further addition of polydadmac (step II, Figure 6) led to the step-by-step neutralization of the negative charges of flocculant included in the primary aggregates, accompanied by rearrangements of chains and the formation of more compact particles with lower sizes. This assumption is supported by the monotonous decrease of the particle sizes found by DLS measurements with an increase of the mass ratio $\mathrm{mg}$ polydadmac/mg flocculant up to about 0.9 .

For the polyion pair investigated in this work, it was observed by both DLS and $\zeta$ potential (Figure 6), that for a dose ranging from 0.9 up to $1.5 \mathrm{mg}$ polydadmac/mg flocculant, the particle size remained almost constant (step III, Figure 6). It seems that the ratio of charge of about 1.7 is critical for these systems, because an abrupt increase of the particle sizes (secondary aggregation) and decrease in $\zeta$ were observed after this ratio (step IV, Figure 6).

Taking acount the results discussed above, NIPECs were prepared at a ratio of $1.5 \mathrm{mg}$ polydadmac/mg flocculant with the following characteristics: particle size $132 \mathrm{~nm}$ and $\zeta=$ $-25 \mathrm{mV}$. The formed complexes were used as a new flocculant in destabilizing the dispersed solids of the wastewater from electroplating process. Figure 7 shows the variation of residual TSS and $\zeta$ in the supernatant according to NIPECs dose $(\mathrm{mg} / \mathrm{L})$. The dispersion of NIPECs is more effective in removing suspended solids and has a wider flocculation window ( $24 \mathrm{mg} / \mathrm{L}$ to $35.6 \mathrm{mg} / \mathrm{L}$ of NIPECs) compared with the pure flocculant. NIPECs dose required (greater than $41 \mathrm{mg} / \mathrm{L}$ ) for the restabilization of dispersed particles, in which the value is $\zeta=-8.6 \mathrm{mV}$, is higher in comparison with the dose of flocculant in system (A) (see above). 


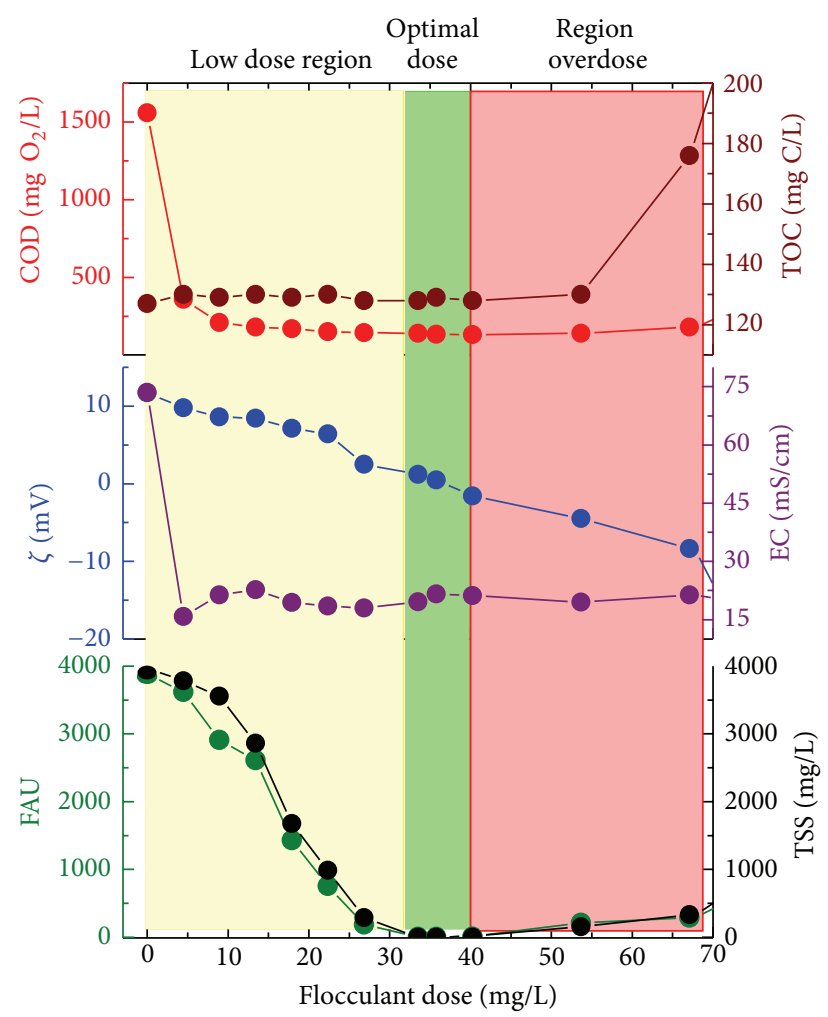

Figure 8: Turbidity, TSS, $\zeta$, EC, COD, and TOC of the supernatant in the single flocculation at $\mathrm{pH} 5$ with flocculant.

At a dose of $6 \mathrm{mg} / \mathrm{L}$ of NIPECs, COD is reduced from 1558 to $360 \mathrm{mg} / \mathrm{L}$ and decreases more in a range between 18 and $35 \mathrm{mg} / \mathrm{L}$ COD, while TOC is kept constant. The lower COD value of $132 \mathrm{mg} / \mathrm{mL}$ is achieved with a dose of $35 \mathrm{mg} / \mathrm{L}$ of NIPECs. At doses greater than $41 \mathrm{mg} / \mathrm{L}$ of NIPECs the restabilization of dispersed solids is induced. Again TOC increased corresponding to the increase in the concentration of residual NIPECs (see Figure 7).

3.5. Single Flocculation at $p H<\operatorname{IEP}(C)$. The residual water at $\mathrm{pH} 5$ has positive $\zeta=11 \mathrm{mV}$. At this $\mathrm{pH}$ flocculant has higher charge density (Figure 2). Figure 8 shows the variation of TSS content in the supernatant as a function of the flocculant dose. It is observed that in the region of overdose, the increase of dispersed solids has a smaller slope compared with the restabilization region of the single flocculation at $\mathrm{pH} 7$ (A). The optimal dose corresponds to $33.5 \mathrm{mg} / \mathrm{L}$ of flocculant, greater than case $(\mathrm{A})$. The flocculation window was between 33.5 and $40 \mathrm{ppm}$ of flocculant.

3.6. Dual Flocculation (D). The residual water at $\mathrm{pH} 9$ has $\zeta=-28 \mathrm{mV}$. Therefore, the addition of flocculant at this $\mathrm{pH}$ has no meaning because the flocculant provokes repulsive interaction that restabilizes particles. Since at $\mathrm{pH} 9$ the maximum cation removal is achieved, it was decided to perform the process of coagulation using the polydadmac.

In this case, the $\zeta$ variation versus polydadmac dose confirms the proper performance of the dual flocculation

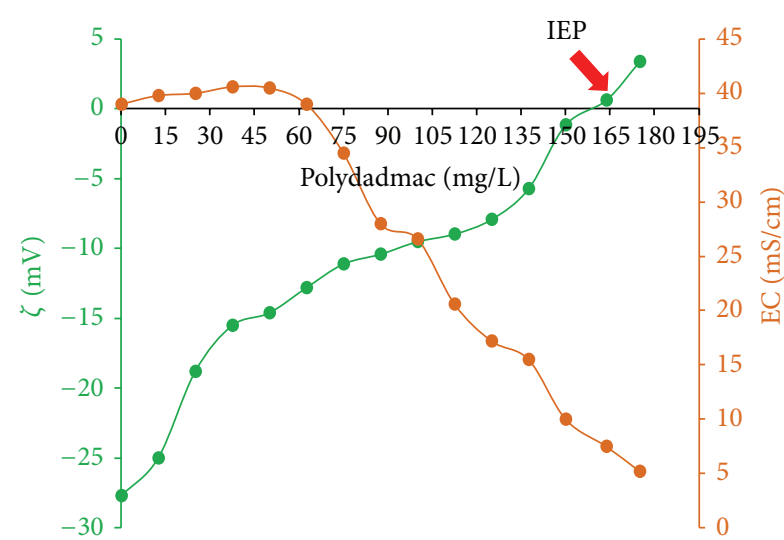

FIGURE 9: Effect of dose of polydadmac in $\zeta$ and EC the supernatant of the wastewater.

process, wherein the optimum condition of the coagulation step is achieved with a dose of $162 \mathrm{mg} / \mathrm{L}$. Complementary, Figure 9 shows the EC and $\zeta$ of the resulting supernatant for each dose of polydadmac. It is noted that the dosage of polydadmac decreases the surface charge of the dispersed particles and the isoelectric point is reached at $162 \mathrm{mg} / \mathrm{L}$ of polydadmac. At doses greater than $162 \mathrm{ppm}$, inversion of surface charge of the dispersed particles was observed. The addition of polydadmac in the coagulation step causes a decrease in the EC; this is attributed to the removal of the anions present in the water that interact with positive sites in the quaternary polydadmac chains (Figure 9).

The next step after finding the optimal dose of polydad$\mathrm{mac}$ is the addition of flocculant. Figure 10 shows that a dose of $3.8 \mathrm{mg} / \mathrm{L}$ of flocculant reduced COD from $1432 \mathrm{mg} / \mathrm{L}$ to $380 \mathrm{mg} / \mathrm{L}$, whereas the TOC remains constant. Increasing flocculant doses progressively reduces the COD. At $18.8 \mathrm{mg} / \mathrm{L}$ TOC decreased to $119 \mathrm{mg} / \mathrm{L}$.

At a dose of $26.4 \mathrm{mg} / \mathrm{L}$ of flocculant, which corresponds to the optimum dose for the removal of TSS, TOC decreased to $115 \mathrm{mg} / \mathrm{L}$. In the range of $26.4 \mathrm{mg} / \mathrm{L}$ to $67 \mathrm{mg} / \mathrm{L}$ of flocculant, TOC decreased markedly; it is noteworthy that in this dosage range metal content decreased to comply with regulations. At the dose of $67 \mathrm{mg} / \mathrm{L}$ the maximum TOC and COD attaint $45 \mathrm{mg} / \mathrm{L}$. The dose of $77 \mathrm{mg} / \mathrm{L}$ of flocculant has an adverse effect on water quality, observed in increasing TSS and cations; this overdose of flocculant causes the increased value of $\zeta, \mathrm{COD}$, and TOC. The increase of COD is mainly attributed to the redispersion of solids corresponding to the inorganic material, while increasing TOC is attributed to increased residual flocculant concentration in solution (organic matter). The coagulation-flocculation process under described conditions removed OM up to $37 \%$.

3.7. Comparison of Flocculation Windows. A resume of all four strategies tested with wastewater from electroplating section in an assembly semiconductor industry is reported in Table 2. Results indicate that flocculation windows are determined not only by $\mathrm{pH}$ value but also by the combination of commercial PE doses and addition sequence. 
TABLE 2: Comparison of the dose and optimal flocculation windows.

\begin{tabular}{lccc}
\hline Treatment system & $\mathrm{pH}$ & Optimal dose (ppm) & Flocculation window (ppm) \\
\hline (A) Single flocculation & 5 & 33.5 & 33.5 to 40.2 \\
(B) Single flocculation & 7 & 15.6 & 15.6 to 20.1 \\
(C) NIPECs flocculation & 7 & 23.7 & 23.7 to 35.6 \\
(D) DUAL coagulation & 9 & 162 & 26.4 to 67 \\
$\quad$ Flocculation & & 26.4 & \\
\hline
\end{tabular}

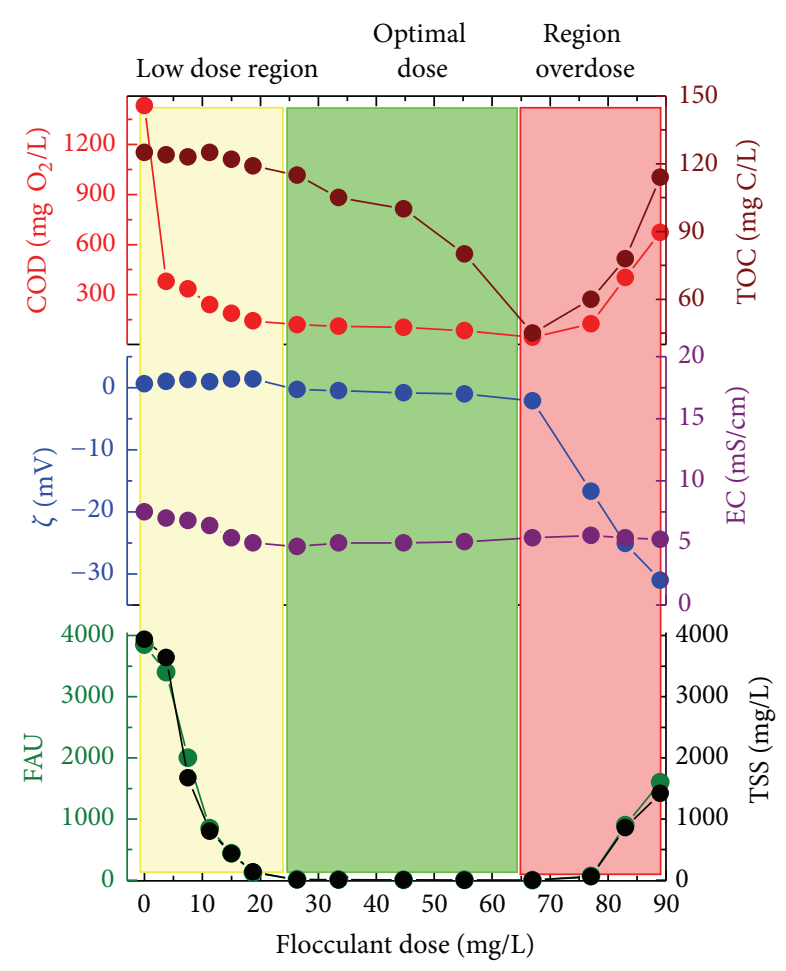

FIgure 10: Turbidity, TSS, $\zeta$, EC, residual COD, and TOC of the supernatant in the dual flocculation at $\mathrm{pH} 9$ using polydadmac and flocculant.

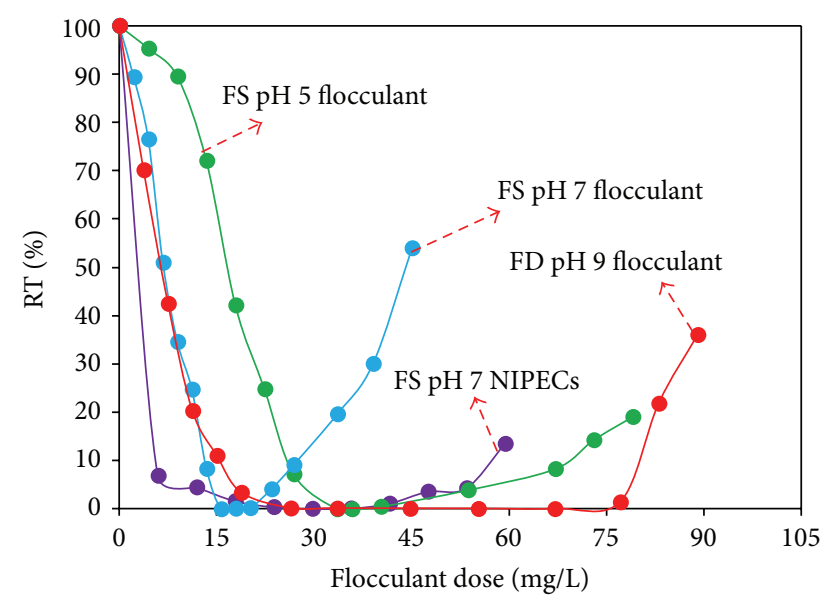

FIGURE 11: Flocculation windows comparison between different systems treatment.
Finally, Figure 11 presents the comparison of all flocculation windows. This representation is useful to select the ranges of flocculant doses under different $\mathrm{pH}$ conditions and additions strategies. Analysis of $\zeta$ potential versus coagulant dosage results is used to evaluate the effectiveness of various polyelectrolytes. Knowledge of $\zeta$ potential is important to adjustment of coagulant dosage levels periodically in order to minimize the cost of chemicals for wastewater treatment.

It was observed that at $\mathrm{pH} 5$ the optimum dose is $33.5 \mathrm{mg} / \mathrm{L}$ and the flocculation dosage window is very short 33.5 to $40.0 \mathrm{mg} / \mathrm{L}$ of flocculant. The single flocculation window at $\mathrm{pH} 7$ using the flocculant is much shorter (15.6 to $20.0 \mathrm{mg} / \mathrm{L}$ ) than at $\mathrm{pH} 5$; the redispersion zone is steeper than flocculation at $\mathrm{pH} 5$. The optimal dose of flocculant at a $\mathrm{pH}$ of 7 is lower by $50 \%$ than that required for the simple flocculation at $\mathrm{pH}$ of 5 . Flocculation window obtained in simple flocculation process at $\mathrm{pH} 7$ using NIPECs is flattened compared to the previous two, and since the first dosage of NIPECs considerably a decreased in turbidity is achieved.

Flocculation window corresponds to NIPECs that is $24.0 \mathrm{mg} / \mathrm{L}$ to $35.6 \mathrm{mg} / \mathrm{L}$, which is much wider than a $\mathrm{pH}$ of 5 and 7 using only the flocculant.

The flocculation window in wastewater treatment by dual flocculation at $\mathrm{pH} 9$ is displaced to higher flocculant dose ( $26.4 \mathrm{mg} / \mathrm{L}$ to $67.0 \mathrm{mg} / \mathrm{L}$ of flocculant). Unlike using NIPECs flocculation window, over $8.1 \mathrm{ppm}$ interpolyelectrolyte complex is required to achieve approximately the same \%RT compared to the optimum dose of the flocculant used in the simple flocculation at $\mathrm{pH} 7$.

\section{Conclusions}

The construction of a $\mathrm{pH}-\zeta$ curve for wastewater, cationic $\mathrm{PE}$, and anionic $\mathrm{PE}$ is the first step previous to selecting PE type and dose in a coagulation-flocculation process as those used in the semiconductor industry. This type of plot allows identifying the isoelectric point for each polyelectrolyte and wastewater. The flocculant has its isoelectric point at a $\mathrm{pH}$ of 2.5 , at which $\mathrm{pH}>$ IEP interacts with the dispersed particles with positive surface charge.

Measurement of COD, TOC, TSS, and turbidity during the coagulation-flocculation windows construction allowed determining the separation efficiencies and the environmental impact. Zeta potential measurements served as a physicochemical evidence of the coagulation-flocculation efficiency in micro-Jars experiments.

At the $\mathrm{pH}$ value of 7, the flocculation window is 15.6 to $20.1 \mathrm{ppm}$ and any higher flocculant dose at $15.6 \mathrm{ppm}$, due 
to the fact that the high charge density of polyelectrolyte has a negative effect on the efficiency of removal of metal hydroxides formed by the restabilization of suspended solids.

The use of the flocculant to $\mathrm{pH} 5$ in the removal of suspended particles is effective but requires a much higher dose (33.5 ppm flocculant) compared to a required $\mathrm{pH}$ of 7 (15.6 ppm flocculant), due to the decrease of the charge density of the flocculant with respect to $\mathrm{pH}$.

The main advantage of NIPECs is the substantial extension of the flocculation window. However, the optimal NIPECs concentration required for flocculation is higher (23-35 ppm NIPECs) than that required when using the flocculant only. The complexes formed in a ratio of $1.5 \mathrm{mg}$ polydadmac/mg flocculant were tested in the destabilization of the dispersion of residual water. The NIPECs flocculants are more effective than single anionic polyelectrolyte; the critical concentration for restabilizing suspended solids is much larger for NIPECs (35 ppm).

The wastewater treatment by dual flocculation at $\mathrm{pH} 9$ (162 ppm corresponding to PIE polydadmac residual water and $67 \mathrm{ppm}$ of flocculant) manages content efficiently to remove turbidity, organic matter, and suspended solids up to $37 \%$.

Every single flocculation system addition (flocculant or NIPECs) had the effect of diminishing COD, achieving $125 \mathrm{mg} \mathrm{O}_{2} / \mathrm{L}$ for the optimal dose of each system. Complete removal of TSS and turbidity is achieved at the optimal dose of polyelectrolyte, while TOC remained roughly constant at $120 \mathrm{mg} \mathrm{C} / \mathrm{L}$.

\section{Conflict of Interests}

The authors declare that there is no conflict of interests regarding the publication of this paper.

\section{Acknowledgments}

Authors acknowledge the enterprise International Rectifiers, Anonymous Society, which through Manuel González, Eng., gave a confident vote to a research group situated in Tijuana to develop a systematic and scientific work that helped in a better operation of their wastewater treatment plant. Authors also thank the Autonomous University of Baja California, Mexico, UABC, that throughout Dr. José Manuel CornejoBravo provide all facilities to use the Zetasizer equipment for zeta potential measurements. Eduardo Alberto LópezMaldonado gives his gratitude to the National Council of Science and Technology (CONACYT) in Mexico, for the fellowship received in his $\mathrm{Ph}$. D. studies.

\section{References}

[1] B. Bolto and J. Gregory, "Organic polyelectrolytes in water treatment," Water Research, vol. 41, no. 11, pp. 2301-2324, 2007.

[2] C. Negro, E. Fuente, L. M. Sánchez, Á. Blanco, and J. Tijero, "Evaluation of an alternative flocculation system for manufacture of fiber-cement composites," Industrial \& Engineering Chemistry Research, vol. 45, no. 20, pp. 6672-6678, 2006.
[3] A. H. Mahvi and M. Razavi, "Application of polyelectrolyte in turbidity removal from surface water," American Journal of Applied Sciences, vol. 2, no. 1, pp. 397-399, 2005.

[4] T. Tripathy and B. Rajan De, "Flocculation: a new way to treat waste water," Journal of Physical Sciences, vol. 10, pp. 93-127, 2006.

[5] S. Dragan, A. Maftuleac, I. Dranca, L. Ghimici, and T. Lupascu, "Flocculation of montmorillonite by some hydrophobically modified polycations containing quaternary ammonium salt groups in the backbone," Journal of Applied Polymer Science, vol. 84, no. 4, pp. 871-876, 2002.

[6] L. Ghimici, I. A. Dinu, and E. S. Dragan, "Application of polyelectrolytes in phase separation processes," in New Trends in Ionic (Co) Polymers and Hybrids, pp. 31-64, Nova Science, Hauppauge, NY, USA, 2007.

[7] D. Zeng, J. Wu, and J. F. Kennedy, "Application of a chitosan flocculant to water treatment," Carbohydrate Polymers, vol. 71, no. 1, pp. 135-139, 2008.

[8] F. Renault, B. Sancey, P.-M. Badot, and G. Crini, "Chitosan for coagulation/flocculation processes-an eco-friendly approach," European Polymer Journal, vol. 45, no. 5, pp. 1337-1348, 2009.

[9] S. Bratskaya, V. Avramenko, S. Schwarz, and I. Philippova, "Enhanced flocculation of oil-in-water emulsions by hydrophobically modified chitosan derivatives," Colloids and Surfaces A: Physicochemical and Engineering Aspects, vol. 275, no. 1-3, pp. 168-176, 2006.

[10] J. R. Rose and M. R. St. John, "Flocculation," in Encyclopedia of Polymer Science and Engineering, J. I. Kroschwitz, Ed., vol. 7, pp. 211-233, John Wiley \& Sons, New York, NY, USA, 2nd edition, 1987.

[11] J. Gregory, "The stability of solid-liquid dispersions in the presence of polymers," in Solid/liquid Dispersions, T. F. Tadros, Ed., pp. 163-181, Academic Press, London, UK, 1987.

[12] C. Laue and D. Hunkeler, "Chitosan-graft-acrylamide polyelectrolytes: synthesis, flocculation, and modeling," Journal of Applied Polymer Science, vol. 102, no. 1, pp. 885-896, 2006.

[13] X. Yu and P. Somasundaran, "Enhanced flocculation withdouble flocculants," Colloids and Surfaces A: Physicochemical and Engineering Aspects, vol. 81, pp. 17-23, 1993.

[14] A. Fan, N. J. Turro, and P. Somasundaran, "A study of dual polymer flocculation," Colloids and Surfaces A: Physicochemical and Engineering Aspects, vol. 162, no. 1-3, pp. 141-148, 2000.

[15] G. Petzold, M. Mende, K. Lunkwitz, S. Schwarz, and H.M. Buchhammer, "Higher efficiency in the flocculation of clay suspensions by using combinations of oppositely charged polyelectrolytes," Colloids and Surfaces A: Physicochemical and Engineering Aspects, vol. 218, no. 1-3, pp. 47-57, 2003.

[16] B. U. Cho, G. Garnier, T. G. M. van de Ven, and M. Perrier, "A bridging model for the effects of a dual component flocculation system on the strength of fiber contacts in flocs of pulp fibers: implications for control of paper uniformity," Colloids and Surfaces A: Physicochemical and Engineering Aspects, vol. 287, no. 1-3, pp. 117-125, 2006.

[17] I. Kashiki and A. Suzuki, "On a new type of flocculant," Industrial \& Engineering Chemistry Fundamentals, vol. 25, no. 1, pp. 120-125, 1986.

[18] A. Suzuki and I. Kashiki, "Flocculation of suspension by binary (polycation-polyanion) flocculant," Industrial \& Engineering Chemistry Research, vol. 26, no. 7, pp. 1464-1468, 1987.

[19] H. M. Buchhammer, G. Petzold, and K. Lunkwitz, "Salt effect on formation and properties of interpolyelectrolyte complexes and 
their interactions with silica particles," Langmuir, vol. 15, no. 12, pp. 4306-4310, 1999.

[20] H.-M. Buchhammer, G. Petzold, and K. Lunkwitz, "Nanoparticles based on polyelectrolyte complexes: effect of structural and net charge on the sorption capability for solved organic molecules," Colloid and Polymer Science, vol. 278, no. 9, pp. 841$847,2000$.

[21] R. S. Nyström, J. B. Rosenholm, and K. Nurmi, "Flocculation of semidilute calcite dispersions induced by anionic sodium polyacrylate-cationic starch complexes," Langmuir, vol. 19, no. 9, pp. 3981-3986, 2003.

[22] G. Petzold, U. Geissler, N. Smolka, and S. Schwarz, "Influence of humic acid on the flocculation of clay," Colloid and Polymer Science, vol. 282, no. 7, pp. 670-676, 2004.

[23] S. Schwarz and E. S. Dragan, "Nonstoichiometric interpolyelectrolyte complexes as colloidal dispersions based on NaPAMPS and their interaction with colloidal silica particles," Macromolecular Symposia, vol. 210, no. 1, pp. 185-191, 2004.

[24] M. Mende, S. Schwarz, G. Petzold, and W. Jaeger, "Destabilization of model silica dispersions by polyelectrolyte complex particles with different charge excess, hydrophobicity, and particle size," Journal of Applied Polymer Science, vol. 103, no. 6, pp. 3776-3784, 2007.

[25] G. Petzold, S. Schwarz, and K. Lunkwitz, "Higher efficiency in particle flocculation by using combinations of oppositely charged polyelectrolytes," Chemical Engineering \& Technology, vol. 26, no. 1, pp. 48-53, 2003.

[26] X. Yu and P. Somasundaran, "Role of polymer conformation in interparticle-bridging dominated flocculation," Journal of Colloid and Interface Science, vol. 177, no. 2, pp. 283-287, 1996.

[27] H. Ono and Y. Deng, "Flocculation and retention of precipitated calcium carbonate by cationic polymeric microparticle flocculants," Journal of Colloid and Interface Science, vol. 188, no. 1, pp. 183-192, 1997.

[28] S. H. Lin and C. D. Kiang, "Combined physical, chemical and biological treatments of wastewater containing organics from a semiconductor plant," Journal of Hazardous Materials, vol. 97, no. 1-3, pp. 159-171, 2003.

[29] P. A. Moussas and A. I. Zouboulis, "A new inorganic-organic composite coagulant, consisting of Polyferric Sulphate (PFS) and Polyacrylamide (PAA)," Water Research, vol. 43, no. 14, pp. 3511-3524, 2009.

[30] Y. Wang, B. Y. Gao, Q. Y. Yue, J. C. Wei, and W. Z. Zhou, "Novel composite flocculent polyferric chloridepolydimethyldiallylammonium chloride (PFC-PDMADAAC): its characterization and flocculation efficiency," Water Practice and Technology, vol. 1, no. 3, pp. 1-9, 2006.

[31] N. D. Tzoupanos and A. I. Zouboulis, "Preparation, characterisation and application of novel composite coagulants for surface water treatment," Water Research, vol. 45, no. 12, pp. 3614-3626, 2011.

[32] H. Tang and B. Shi, "The characteristics of composite flocculants synthesized with polyaluminium and organic polymers, chemical water and wastewater treatment VII," in Proceedings of the 10th Gothenburg Symposium, H. H. Hahn and E. Hoffmann, Eds., pp. 17-28, Gothenburg, Sweden, 2002.

[33] W. Y. Yang, J. W. Qian, and Z. Q. Shen, "A novel flocculant of $\mathrm{Al}(\mathrm{OH})_{3}$-polyacrylamide ionic hybrid," Journal of Colloid and Interface Science, vol. 273, no. 2, pp. 400-405, 2004.
[34] K. E. Lee, T. T. Teng, N. Morad, B. T. Poh, and Y. F. Hong, "Flocculation of kaolin in water using novel calcium chloridepolyacrylamide $\left(\mathrm{CaCl}_{2}-\mathrm{PAM}\right)$ hybrid polymer," Separation and Purification Technology, vol. 75, no. 3, pp. 346-351, 2010. 

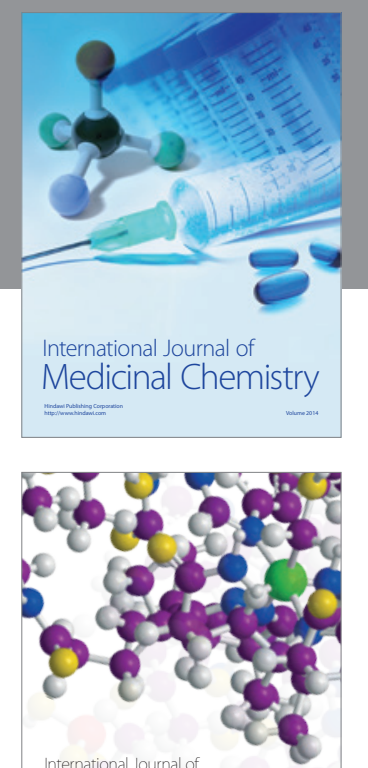

\section{Carbohydrate} Chemistry

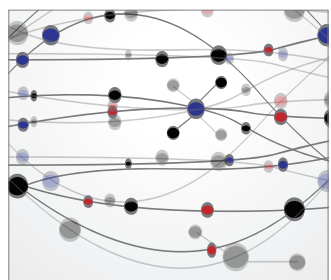

The Scientific World Journal
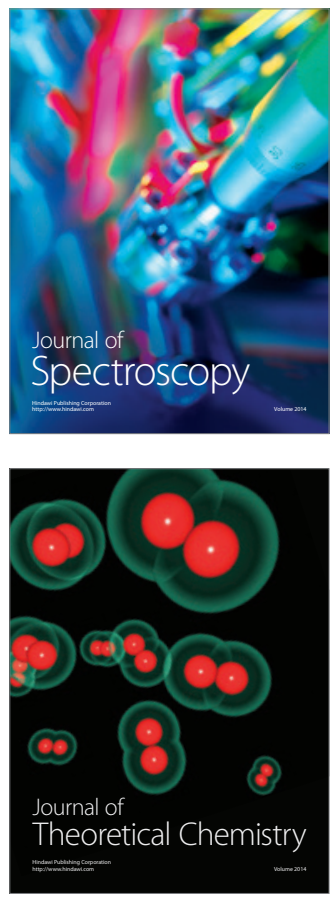
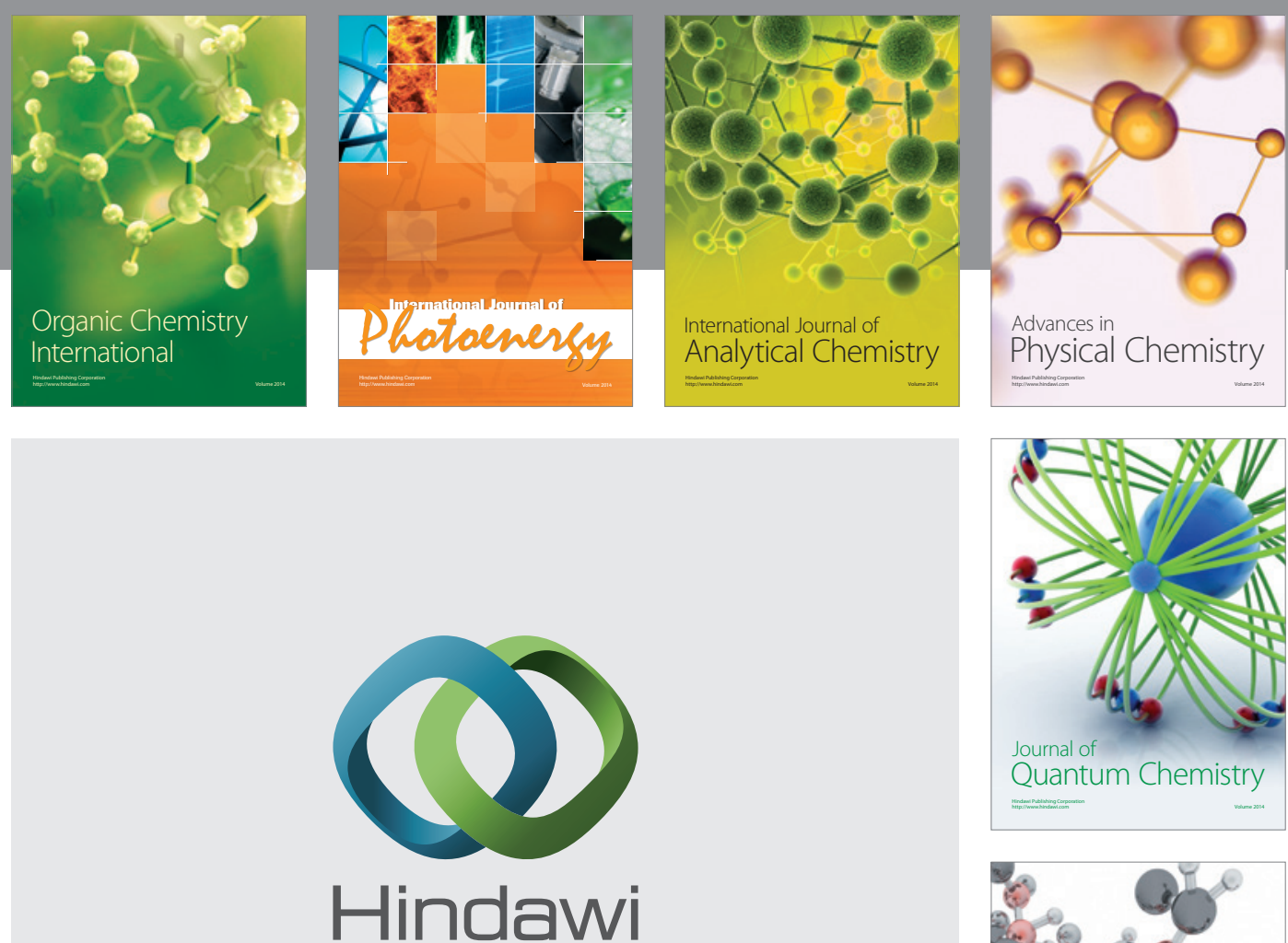

Submit your manuscripts at

http://www.hindawi.com

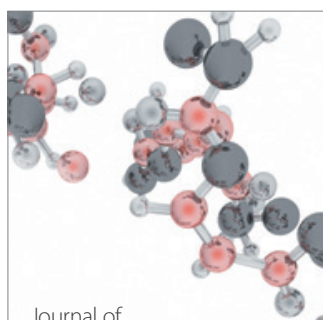

Analytical Methods

in Chemistry

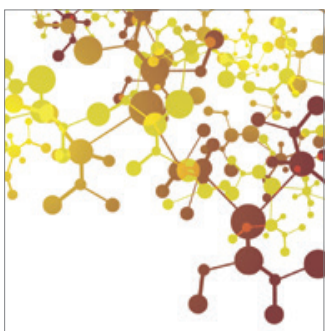

Journal of

Applied Chemistry

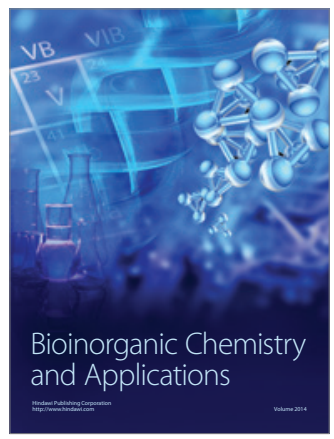

Inorganic Chemistry
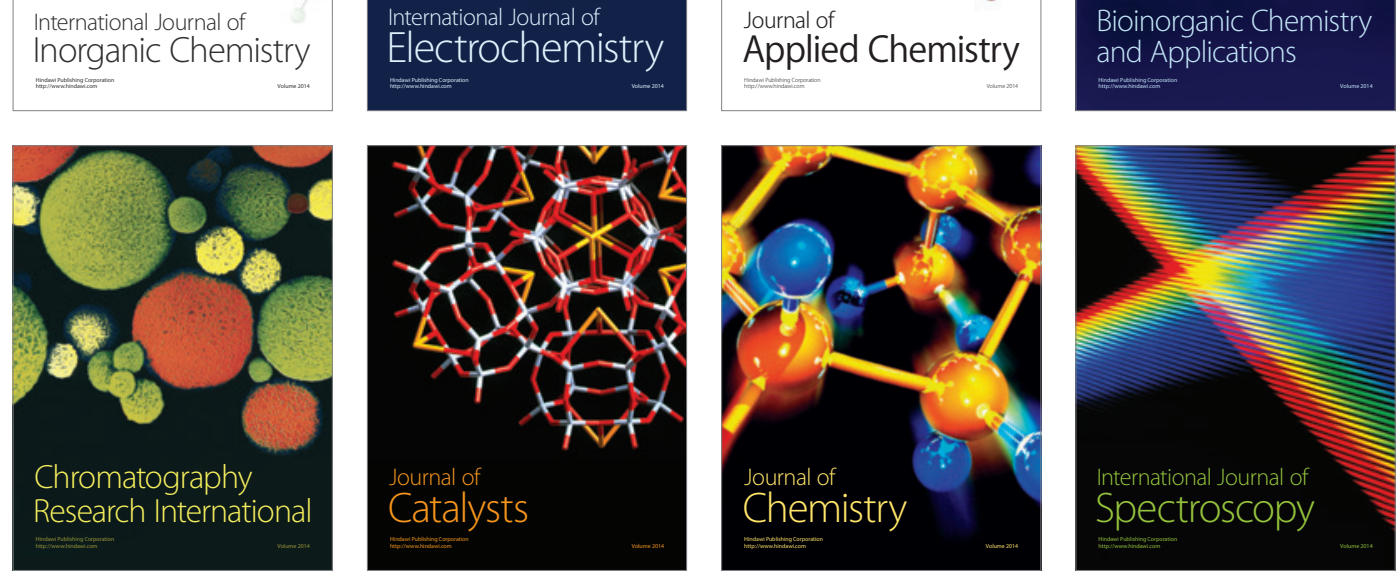